NCSI have a higher mortality and suffer more serious neurologic injury than with single level injury. Injured spinal segments occur at a greater distance in patients with neurologic injury or death. Younger patients with NCSIs had a high rate of associated injuries. Clinicians must be aware of the incidence of NCSIs in children, as well as their associations.

\title{
0688 THE EPIDEMIOLOGY AND CLINICAL FEATURES OF MULTIPLE, NON-CONTIGUOUS SPINE INJURIES IN CHILDREN
}

P J Moroz*, S Kingwell Correspondence: University of Ottawa/Children's Hospital of Eastern Ontario, Department of Surgery, Children's Hospital of Eastern Ontario 401 Smyth Road, Ottawa, Ontario K1H8L1, Canada

\subsection{6/ip.2010.029215.688}

Multiple - non-contiguous spinal injuries (NCSI) are complex injuries frequently missed and with increased potential for adverse outcomes. Adult incidence is reported as $1.6-34 \%$, with greater risk of mortality and fracture instability; the paediatric incidence is unknown. A retrospective review at an academic paediatric trauma centre over a 15 year period identified $25(11.8 \%)$ out of 211 patients with NCSI, with a mean age of 10.7 years. MVC was the primary cause for ages $0-9$, while a fall was seen for ages 10-17. The mean number of vertebral levels injured was 3.2. The most common region was the thoracic spine, with a mean of 5.4 (range 1-22) intact vertebral levels between injuries. Twenty-four percent with NCSI had a neurologic deficit versus $9.7 \%$ with single level, contiguous injuries ( $R R 2.48(1.09,5.65))$. Seven $(78 \%)$ of nine patients aged 0-9 suffered an associated injury, usually a visceral injury. Mortality was $8.0 \%$ in patients with NCSI versus $2.7 \%$ in patients with single level or contiguous injury. Children with 